\title{
Use of evidence in health inequalities policy
}

\author{
${ }^{1} \mathrm{M}$ Tsagalidou, ${ }^{2} \mathrm{CM}$ Jones \\ ${ }^{1}$ Project Officer; ${ }^{2}$ Consultant, Public Health Sciences, NHS Health Scotland, Edinburgh, UK
}

The Public Health symposium was held on 8 May 2014 at the Royal College of Physicians of Edinburgh

DECLARATION OF INTERESTS No conflict of interests declared.

\author{
Correspondence to M Tsagalidou \\ Public Health Sciences \\ NHS Health Scotland \\ I South Gyle Crescent \\ Edinburgh EHI2 9EB \\ UK
}

e-mail melanie.tsagalidou@nhs.net

\section{INTRODUCTION}

Addressing health inequalities has been a key theme of recent Scottish governments; however, since the 1980s, these inequalities have been getting worse. The research evidence from the Marmot Review clearly shows that the redistribution of power, wealth and resources across society is required to reverse these worsening trends, yet policies to make effective changes and achieve greater equality in Scotland are still to be implemented. Using real life examples, experiential knowledge and the latest research evidence, this symposium explored the reasons for the lack of progress in addressing inequalities and points to a way forward.

\section{SESSION I: DEFINING THE ISSUES}

The first session was chaired by Dr Colwyn Jones (Consultant in Dental Public Health, NHS Health Scotland) with talks by Professor Mark Petticrew (London School of Hygiene and Tropical Medicine), Professor John Frank (Scottish Collaboration for Public Health Research and Policy) and Dr Andrew Fraser (NHS Health Scotland). All highlighted the difficulty in using evidence to influence national policy. The shortterm political cycle makes longer term interventions hard for politicians to accept and implement. Researchers need to be familiar with cultures of accepting, manipulating and using evidence and they have to understand the types of evidence that would be useful to support politicians when making decisions. If not politically active then, as a minimum, public health must be politically aware.

\section{SESSION 2: INDUSTRY AND ADVOCACY}

Professor Jeff Collin, (University of Edinburgh) and Dr Jim McCambridge (London School of Hygiene and Tropical Medicine) addressed the tactics of undermining evidence by the tobacco and alcohol industries around effective public health actions in a session chaired by Professor Carol Tannahill (Glasgow Centre for Population Health). One example was how plain cigarette packaging was stopped by tobacco industry lobbying of the UK government. Another was the use of a bogus harm reduction strategy by the drinks industry which played an active role in watering down public health policy formation, by strategically placing themselves as stakeholders. Dr McCambridge concluded that researchers who want to understand and influence policy formation need to take all of the industry reactions into account. Dr Katherine Smith (University of Edinburgh) emphasised that to support uptake and use of research, researchers need to go beyond publication and advocate their own research to start to bridge the gap between evidence and policy. There was an interactive session which cleverly illustrated the gap between evidence and action, even within a public health audience.

\section{SESSION 3: EVIDENCE, MEDIA AND HEALTH}

Professor Laurence Moore (MRC/SCO Social and Public Health Sciences Unit, University of Glasgow) addressed the challenges of making evidence more useful standardised interventions were often not replicable in the real world and the need to shift focus from interventions to creating conditions for change. He contrasted clinical research with public health research practices: in the first, the evidence generation and use is integral to training. However, public health policies are often poorly evidenced and under-evaluated. To move forward we need investment in structures and processes that facilitate knowledge sharing between all the stakeholders of evidence such as researchers, decisionmakers, practitioners and the public. 
Speaking as an ex-civil servant, Sir Harry Burns (Strathclyde University) stressed that the role of the civil servant is to advise. Some policies are inherited from previous governments and they may not fit together with new initiatives. Politicians are accountable for decisions, but there is tension between central and local government and rivalry between local councils. If any change is to be accepted the key stakeholders need to be involved in the process. He mentioned the Early Years Collaborative as an example of where learning has been fed back and informed different courses of action.

The inequalities landscape can change if the public become advocates of the Scotland it aspires to live in, by focusing and building on the positives rather than criticise the things that aren't working. Use appropriate methods of evaluation for complex systems. Scientific rigour is important, but may need to be re-defined. Economic debate and its relation with inequalities should be considered in addition to everything that had gone before.

The final speaker, Mr Alex Bell, reflected on his experiences as Head of Policy to the Scottish Nationalist Party for two years. In his view, Scotland faces global problems requiring global answers, such as on health inequalities. We require a lead on changes, but public servants don't have a clear steer about the direction of these changes from the citizens.
He suggested that public servants are interested in evidence only when something is highlighted as inefficient in the press. Such press interest makes it more difficult for policymakers to legislate and continue a course of action.

\section{TAKE-HOME MESSAGE}

The lack of a linear relationship between politics and research evidence is clear, with chance, existing networks, serendipity and opportunity playing their part. These are interdependent and could benefit from improving the communication and understanding of each other's needs. Public health professionals must be prepared to make compromises but also be clear in what they are advocating and advising the policymakers so that they in turn will be able to create policies that address the key issues creating health and other inequalities. 